\title{
HSU-ROBBINS AND SPITZER'S THEOREMS FOR THE VARIATIONS OF FRACTIONAL BROWNIAN MOTION
}

\author{
CIPRIAN A. TUDOR \\ SAMOS/MATISSE, Centre d'Economie de La Sorbonne, Université de Panthéon-Sorbonne Paris 1, 90, \\ rue de Tolbiac, 75634 Paris Cedex 13, France \\ email: tudor@univ-paris1.fr
}

Submitted February 16, 2009, accepted in final form June 28, 2009

AMS 2000 Subject classification: 60G15, 60H05, 60F05, 60H07.

Keywords: multiple stochastic integrals, selfsimilar processes, fractional Brownian motion, Hermite processes, limit theorems, Stein's method.

\section{Abstract}

Using recent results on the behavior of multiple Wiener-Itô integrals based on Stein's method, we prove Hsu-Robbins and Spitzer's theorems for sequences of correlated random variables related to the increments of the fractional Brownian motion.

\section{Introduction}

A famous result by Hsu and Robbins [7] says that if $X_{1}, X_{2}, \ldots$ is a sequence of independent identically distributed random variables with zero mean and finite variance and $S_{n}:=X_{1}+\ldots+X_{n}$, then

$$
\sum_{n \geq 1} P\left(\left|S_{n}\right|>\varepsilon n\right)<\infty
$$

for every $\varepsilon>0$. Later, Erdös ([3], [4]) showed that the converse implication also holds, namely if the above series is finite for every $\varepsilon>0$ and $X_{1}, X_{2}, \ldots$ are independent and identically distributed, then $\mathbf{E} X_{1}=0$ and $\mathbf{E} X_{1}^{2}<\infty$. Since then, many authors extended this result in several directions. Spitzer's showed in [13] that

$$
\sum_{n \geq 1} \frac{1}{n} P\left(\left|S_{n}\right|>\varepsilon n\right)<\infty
$$

for every $\varepsilon>0$ if and only if $\mathbf{E} X_{1}=0$ and $\mathbf{E}\left|X_{1}\right|<\infty$. Also, Spitzer's theorem has been the object of various generalizations and variants. One of the problems related to the Hsu-Robbins' and Spitzer's theorems is to find the precise asymptotic as $\varepsilon \rightarrow 0$ of the quantities $\sum_{n \geq 1} P\left(\left|S_{n}\right|>\varepsilon n\right)$ and $\sum_{n \geq 1} \frac{1}{n} P\left(\left|S_{n}\right|>\varepsilon n\right)$. Heyde [5] showed that

$$
\lim _{\varepsilon \rightarrow 0} \varepsilon^{2} \sum_{n \geq 1} P\left(\left|S_{n}\right|>\varepsilon n\right)=\mathbf{E} X_{1}^{2}
$$


whenever $\mathbf{E} X_{1}=0$ and $\mathbf{E} X_{1}^{2}<\infty$. In the case when $X$ is attracted to a stable distribution of exponent $\alpha>1$, Spataru [12] proved that

$$
\lim _{\varepsilon \rightarrow 0} \frac{1}{-\log \varepsilon} \sum_{n \geq 1} \frac{1}{n} P\left(\left|S_{n}\right|>\varepsilon n\right)=\frac{\alpha}{\alpha-1} .
$$

The purpose of this paper is to prove Hsu-Robbins and Spitzer's theorems for sequences of correlated random variables, related to the increments of fractional Brownian motion, in the spirit of [5] or [12]. Recall that the fractional Brownian motion $\left(B_{t}^{H}\right)_{t \in[0,1]}$ is a centered Gaussian process with covariance function $R^{H}(t, s)=\mathrm{E}\left(B_{t}^{H} B_{s}^{H}\right)=\frac{1}{2}\left(t^{2 H}+s^{2 H}-|t-s|^{2 H}\right)$. It can be also defined as the unique self-similar Gaussian process with stationary increments. Concretely, in this paper we will study the behavior of the tail probabilities of the sequence

$$
V_{n}=\sum_{k=0}^{n-1} H_{q}\left(n^{H}\left(B_{\frac{k+1}{n}}-B_{\frac{k}{n}}\right)\right)
$$

where $B$ is a fractional Brownian motion with Hurst parameter $H \in(0,1)$ (in the sequel we will omit the superscript $H$ for $B$ ) and $H_{q}$ is the Hermite polynomial of degree $q \geq 1$ given by $H_{q}(x)=(-1)^{q} e^{\frac{x^{2}}{2}} \frac{d^{q}}{d x^{q}}\left(e^{-\frac{x^{2}}{2}}\right)$. The sequence $V_{n}$ behaves as follows (see e.g. [9], Theorem 1; the result is also recalled in Section 3 of our paper): if $0<H<1-\frac{1}{2 q}$, a central limit theorem holds for the renormalized sequence $Z_{n}^{(1)}=\frac{V_{n}}{c_{1, q, H} \sqrt{n}}$ while if $1-\frac{1}{2 q}<H<1$, the sequence $Z_{n}^{(2)}=\frac{V_{n}}{c_{2, q, H} n^{1-q(1-H)}}$ converges in $L^{2}(\Omega)$ to a Hermite random variable of order $q$ (see Section 2 for the definition of the Hermite random variable and Section 3 for a rigorous statement concerning the convergence of $V_{n}$ ). Here $c_{1, q, H}, c_{2, q, H}$ are explicit positive constants depending on $q$ and $H$.

We note that the techniques generally used in the literature to prove the Hsu-Robbins and Spitzer's results are strongly related to the independence of the random variables $X_{1}, X_{2}, \ldots$ In our case the variables are correlated. Indeed, for any $k, l \geq 1$ we have

$$
\mathbf{E}\left(H_{q}\left(B_{k+1}-B_{k}\right) H_{q}\left(B_{l+1}-B_{l}\right)\right)=\frac{1}{(q !)^{2}} \rho_{H}(k-l)
$$

where the correlation function is $\rho_{H}(k)=\frac{1}{2}\left((k+1)^{2 H}+(k-1)^{2 H}-2 k^{2 H}\right)$ which is not equal to zero unless $H=\frac{1}{2}$ (which is the case of the standard Brownian motion). We use new techniques based on the estimates for the multiple Wiener-Itô integrals obtained in [2], [10] via Stein's method and Malliavin calculus. Concretely, we study in this paper the behavior as $\varepsilon \rightarrow 0$ of the quantities

$$
\sum_{n \geq 1} \frac{1}{n} P\left(V_{n}>\varepsilon n\right)=\sum_{n \geq 1} \frac{1}{n} P\left(Z_{n}^{(1)}>c_{1, q, H}^{-1} \varepsilon \sqrt{n}\right)
$$

and

$$
\sum_{n \geq 1} P\left(V_{n}>\varepsilon n\right)=\sum_{n \geq 1} P\left(Z_{n}^{(1)}>c_{1, q, H}^{-1} \varepsilon \sqrt{n}\right),
$$

if $0<H<1-\frac{1}{2 q}$ and of

$$
\sum_{n \geq 1} \frac{1}{n} P\left(V_{n}>\varepsilon n^{2-2 q(1-H)}\right)=\sum_{n \geq 1} \frac{1}{n} P\left(Z_{n}^{(2)}>c_{2, q, H}^{-1} \varepsilon n^{1-q(1-H)}\right)
$$


and

$$
\sum_{n \geq 1} P\left(V_{n}>\varepsilon n^{2-2 q(1-H)}\right)=\sum_{n \geq 1} P\left(Z_{n}^{(2)}>c_{2, q, H}^{-1} \varepsilon n^{1-q(1-H)}\right)
$$

if $1-\frac{1}{2 q}<H<1$. The basic idea in our proofs is that, if we replace $Z_{n}^{(1)}$ and $Z_{n}^{(2)}$ by their limits (standard normal random variable or Hermite random variable) in the above expressions, the behavior as $\varepsilon \rightarrow 0$ can be obtained by standard calculations. Then we need to estimate the difference between the tail probabilities of $Z_{n}^{(1)}, Z_{n}^{(2)}$ and the tail probabilities of their limits. To this end, we will use the estimates obtained in [2], [10] via Malliavin calculus and we are able to prove that this difference converges to zero in all cases. We obtain that, as $\varepsilon \rightarrow 0$, the quantities (4) and (6) are of order of $\log \varepsilon$ while the functions (5) and (7) are of order of $\varepsilon^{2}$ and $\varepsilon^{1-q(1-H)}$ respectively.

The paper is organized as follows. Section 2 contains some preliminaries on the stochastic analysis on Wiener chaos. In Section 3 we prove the Spitzer's theorem for the variations of the fractional Brownian motion while Section 4 is devoted to the Hsu-Robbins theorem for this sequence.

Throughout the paper we will denote by $c$ a generic strictly positive constant which may vary from line to line (and even on the same line).

\section{Preliminaries}

Let $\left(W_{t}\right)_{t \in[0,1]}$ be a classical Wiener process on a standard Wiener space $(\Omega, \mathscr{F}, \mathbf{P})$. If $f \in L^{2}\left([0,1]^{n}\right)$ with $n \geq 1$ integer, we introduce the multiple Wiener-Itô integral of $f$ with respect to $W$. The basic reference is $[11]$.

Let $f \in \mathscr{S}_{m}$ be an elementary function with $m$ variables that can be written as

$$
f=\sum_{i_{1}, \ldots, i_{m}} c_{i_{1}, \ldots i_{m}} 1_{A_{i_{1}} \times \ldots \times A_{i_{m}}}
$$

where the coefficients satisfy $c_{i_{1}, \ldots i_{m}}=0$ if two indices $i_{k}$ and $i_{l}$ are equal and the sets $A_{i} \in \mathscr{B}([0,1])$ are disjoint. For such a step function $f$ we define

$$
I_{m}(f)=\sum_{i_{1}, \ldots, i_{m}} c_{i_{1}, \ldots i_{m}} W\left(A_{i_{1}}\right) \ldots W\left(A_{i_{m}}\right)
$$

where we put $W(A)=\int_{0}^{1} 1_{A}(s) d W_{s}$ if $A \in \mathscr{B}([0,1])$. It can be seen that the mapping $I_{n}$ constructed above from $\mathscr{S}_{m}$ to $L^{2}(\Omega)$ is an isometry on $\mathscr{S}_{m}$, i.e.

$$
\mathbf{E}\left[I_{n}(f) I_{m}(g)\right]=n !\langle f, g\rangle_{L^{2}\left([0,1]^{n}\right)} \text { if } m=n
$$

and

$$
\mathbf{E}\left[I_{n}(f) I_{m}(g)\right]=0 \text { if } m \neq n .
$$

Since the set $\mathscr{S}_{n}$ is dense in $L^{2}\left([0,1]^{n}\right)$ for every $n \geq 1$ the mapping $I_{n}$ can be extended to an isometry from $L^{2}\left([0,1]^{n}\right)$ to $L^{2}(\Omega)$ and the above properties hold true for this extension.

We will need the following bound for the tail probabilities of multiple Wiener-Itô integrals (see [8], Theorem 4.1)

$$
P\left(\left|I_{n}(f)\right|>u\right) \leq c \exp \left(\left(\frac{-c u}{\sigma}\right)^{\frac{2}{n}}\right)
$$


for all $u>0, n \geq 1$, with $\sigma=\|f\|_{L^{2}\left([0,1]^{n}\right)}$.

The Hermite random variable of order $q \geq 1$ that appears as limit in Theorem 1 , point ii. is defined as (see [9])

$$
Z=d(q, H) I_{q}(L)
$$

where the kernel $L \in L^{2}\left([0,1]^{q}\right)$ is given by

$$
L\left(y_{1}, \ldots, y_{q}\right)=\int_{y_{1} \vee \ldots \vee y_{q}}^{1} \partial_{1} K^{H}\left(u, y_{1}\right) \ldots \partial_{1} K^{H}\left(u, y_{q}\right) d u .
$$

The constant $d(q, H)$ is a positive normalizing constant that guarantees that $\mathbf{E} Z^{2}=1$ and $K^{H}$ is the standard kernel of the fractional Brownian motion (see [11], Section 5). We will not need the explicit expression of this kernel. Note that the case $q=1$ corresponds to the fractional Brownian motion and the case $q=2$ corresponds to the Rosenblatt process.

\section{Spitzer's theorem}

Let us start by recalling the following result on the convergence of the sequence $V_{n}$ (3) (see [9], Theorem 1).

Theorem 1. Let $q \geq 2$ an integer and let $\left(B_{t}\right)_{t \geq 0}$ a fractional Brownian motion with Hurst parameter $H \in(0,1)$. Then, with some explicit positive constants $c_{1, q, H}, c_{2, q, H}$ depending only on $q$ and $H$ we have

i. If $0<H<1-\frac{1}{2 q}$ then

$$
\frac{V_{n}}{c_{1, q, H} \sqrt{n}} \stackrel{\text { Law }}{\longrightarrow}{ }_{n \rightarrow \infty} N(0,1)
$$

ii. If $1-\frac{1}{2 q}<H<1$ then

$$
\frac{V_{n}}{c_{2, q, H} n^{1-q(1-H)}}{\stackrel{\mathrm{L}^{2}}{\longrightarrow}}_{n \rightarrow \infty} Z
$$

where $Z$ is a Hermite random variable given by (10).

In the case $H=1-\frac{1}{2 q}$ the limit is still Gaussian but the normalization is different. However we will not treat this case in the present work.

We set

$$
Z_{n}^{(1)}=\frac{V_{n}}{c_{1, q, H} \sqrt{n}}, \quad Z_{n}^{(2)}=\frac{V_{n}}{c_{2, q, H} n^{1-q(1-H)}}
$$

with the constants $c_{1, q, H}, c_{2, q, H}$ from Theorem 1 .

Let us denote, for every $\varepsilon>0$,

$$
f_{1}(\varepsilon)=\sum_{n \geq 1} \frac{1}{n} P\left(V_{n}>\varepsilon n\right)=\sum_{n \geq 1} \frac{1}{n} P\left(Z_{n}^{(1)}>c_{1, q, H}^{-1} \varepsilon \sqrt{n}\right)
$$

and

$$
f_{2}(\varepsilon)=\sum_{n \geq 1} \frac{1}{n} P\left(V_{n}>\varepsilon n^{2-2 q(1-H)}\right)=\sum_{n \geq 1} \frac{1}{n} P\left(Z_{n}^{(2)}>c_{2, q, H}^{-1} \varepsilon n^{1-q(1-H)}\right)
$$


Remark 1. It is natural to consider the tail probability of order $n^{2-2 q(1-H)}$ in (15) because the $L^{2}$ norm of the sequence $V_{n}$ is in this case of order $n^{1-q(1-H)}$.

We are interested to study the behavior of $f_{i}(\varepsilon)(i=1,2)$ as $\varepsilon \rightarrow 0$. For a given random variable $X$, we set $\Phi_{X}(z)=1-P(X<z)+P(X<-z)$.

The first lemma gives the asymptotics of the functions $f_{i}(\epsilon)$ as $\varepsilon \rightarrow 0$ when $Z_{n}^{(i)}$ are replaced by their limits.

Lemma 1. Consider $c>0$.

i. Let $Z^{(1)}$ be a standard normal random variable. Then as

$$
\frac{1}{-\log c \varepsilon} \sum_{n \geq 1} \frac{1}{n} \Phi_{Z^{(1)}}(c \varepsilon \sqrt{n}) \rightarrow_{\varepsilon \rightarrow 0} 2 .
$$

ii. Let $Z^{(2)}$ be a Hermite random variable or order $q$ given by (10). Then, for any integer $q \geq 1$

$$
\frac{1}{-\log c \varepsilon} \sum_{n \geq 1} \frac{1}{n} \Phi_{Z^{(2)}}\left(c \varepsilon n^{1-q(1-H)}\right) \rightarrow_{\varepsilon \rightarrow 0} \frac{1}{1-q(1-H)} .
$$

Proof: The case when $Z^{(1)}$ follows the standard normal law is hidden in [12]. We will give the ideas of the proof. We can write (see [12])

$$
\sum_{n \geq 1} \frac{1}{n} \Phi_{Z^{(1)}}(c \varepsilon \sqrt{n})=\int_{1}^{\infty} \frac{1}{x} \Phi_{Z^{(1)}}(c \varepsilon \sqrt{x}) d x-\frac{1}{2} \Phi_{Z^{(1)}}(c \varepsilon)-\int_{1}^{\infty} P_{1}(x) d\left[\frac{1}{x} \Phi_{Z^{(1)}}(c \varepsilon \sqrt{x})\right] .
$$

with $P_{1}(x)=[x]-x+\frac{1}{2}$. Clearly as $\varepsilon \rightarrow 0, \frac{1}{\log \varepsilon} \Phi_{Z^{(1)}}(c \varepsilon) \rightarrow 0$ because $\Phi_{Z^{(1)}}$ is a bounded function and concerning the last term it is also trivial to observe that

$$
\begin{aligned}
& \frac{1}{-\log c \varepsilon} \int_{1}^{\infty} P_{1}(x) d\left[\frac{1}{x} \Phi_{Z^{(1)}}(c \varepsilon \sqrt{x})\right] \\
& =\frac{1}{-\log c \varepsilon}\left(-\int_{1}^{\infty} P_{1}(x)\left(\frac{1}{x^{2}} \Phi_{Z^{(1)}}(c \varepsilon \sqrt{x}) d x+c \varepsilon \frac{1}{2} x^{-\frac{1}{2}} \frac{1}{x} \Phi_{Z^{(1)}}^{\prime}(\varepsilon \sqrt{x})\right) d x\right) \rightarrow_{\varepsilon \rightarrow 0} 0
\end{aligned}
$$

since $\Phi_{Z^{(1)}}$ and $\Phi_{Z^{(1)}}^{\prime}$ are bounded. Therefore the asymptotics of the function $f_{1}(\varepsilon)$ as $\varepsilon \rightarrow 0$ will be given by $\int_{1}^{\infty} \frac{1}{x} \Phi_{Z^{(1)}}(c \varepsilon \sqrt{x}) d x$. By making the change of variables $c \varepsilon \sqrt{x}=y$, we get

$$
\lim _{\varepsilon \rightarrow 0} \frac{1}{-\log c \varepsilon} \int_{1}^{\infty} \frac{1}{x} \Phi_{Z^{(1)}}(c \varepsilon \sqrt{x}) d x=\lim _{\varepsilon \rightarrow 0} \frac{1}{-\log c \varepsilon} 2 \int_{c \varepsilon}^{\infty} \frac{1}{y} \Phi_{Z^{(1)}}(y) d y=\lim _{\varepsilon \rightarrow 0} 2 \Phi_{Z^{(1)}}(c \varepsilon)=2 .
$$

Let us consider now the case of the Hermite random variable. We will have as above

$$
\begin{aligned}
& \lim _{\varepsilon \rightarrow 0} \frac{1}{-\log c \varepsilon} \sum_{n \geq 1} \frac{1}{n} \Phi_{Z^{(2)}}\left(c \varepsilon n^{1-q(1-H)}\right) \\
= & \lim _{\varepsilon \rightarrow 0} \frac{1}{-\log c \varepsilon}\left(\int_{1}^{\infty} \frac{1}{x} \Phi_{Z^{(2)}}\left(c \varepsilon x^{1-q(1-H)}\right) d x-\int_{1}^{\infty} P_{1}(x) d\left[\frac{1}{x} \Phi_{Z^{(2)}}\left(c \varepsilon x^{1-q(1-H)}\right)\right]\right)
\end{aligned}
$$


By making the change of variables $c \varepsilon x^{1-q(1-H)}=y$ we will obtain

$$
\begin{aligned}
& \lim _{\varepsilon \rightarrow 0} \frac{1}{-\log c \varepsilon} \int_{1}^{\infty} \frac{1}{x} \Phi_{Z^{(2)}}\left(c \varepsilon x^{1-q(1-H)}\right) d x \\
= & \lim _{\varepsilon \rightarrow 0} \frac{1}{-\log c \varepsilon} \frac{1}{1-q(1-H)} \int_{c \varepsilon}^{\infty} \frac{1}{y} \Phi_{Z^{(2)}}(y) d y=\lim _{\varepsilon \rightarrow 0} \frac{1}{1-q(1-H)} \Phi_{Z^{(2)}}(c \varepsilon)=\frac{1}{1-q(1-H)}
\end{aligned}
$$

where we used the fact that $\Phi_{Z^{(2)}}(y) \leq y^{-2} \mathbf{E}\left|Z^{(2)}\right|^{2}$ and so $\lim _{y \rightarrow \infty} \log y \Phi_{Z^{(2)}}(y)=0$.

It remains to show that $\frac{1}{-\log c \varepsilon} \int_{1}^{\infty} P_{1}(x) d\left[\frac{1}{x} \Phi_{Z^{(2)}}\left(c \varepsilon x^{1-q(1-H)}\right)\right]$ converges to zero as $\varepsilon$ tends to 0 (note that actually it follows from a result by [1] that a Hermite random variable has a density, but we don't need it explicitly, we only use the fact that $\Phi_{Z^{(2)}}$ is differentiable almost everywhere). This is equal to

$$
\begin{aligned}
& \lim _{\varepsilon} \frac{1}{-\log c \varepsilon} \int_{1}^{\infty} P_{1}(x) c \varepsilon(1-q(1-H)) x^{-q(1-H)-1} \Phi_{Z^{(2)}}^{\prime}\left(c \varepsilon x^{1-q(1-H)}\right) d x \\
= & c \frac{\varepsilon}{-\log \varepsilon}(c \varepsilon)^{\frac{q(1-H)}{1-q(1-H)}} \int_{c \varepsilon}^{\infty} P_{1}\left(\left(\frac{y}{c \varepsilon}\right)^{\frac{1}{1-q(1-H)}}\right) \Phi_{Z^{(2)}}^{\prime}(y) y^{-\frac{1}{1-q(1-H)}} d y \\
\leq & c \frac{1}{-\log \varepsilon} \int_{c \varepsilon}^{\infty} P_{1}\left(\left(\frac{1}{c \varepsilon}\right)^{\frac{1}{1-q(1-H)}}\right) \Phi_{Z^{(2)}}^{\prime}(y) d y
\end{aligned}
$$

which clearly goes to zero since $P_{1}$ is bounded and $\int_{0}^{\infty} \Phi_{Z^{(2)}}^{\prime}(y) d y=1$.

The next result estimates the limit of the difference between the functions $f_{i}(\varepsilon)$ given by (14), (15) and the sequence in Lemma 1.

Proposition 1. Let $q \geq 2$ and $c>0$.

i. If $H<1-\frac{1}{2 q}$, let $Z_{n}^{(1)}$ be given by (13) and let $Z^{(1)}$ be standard normal random variable. Then it holds

$$
\frac{1}{-\log c \varepsilon}\left[\sum_{n \geq 1} \frac{1}{n} P\left(\left|Z_{n}^{(1)}\right|>c \varepsilon \sqrt{n}\right)-\sum_{n \geq 1} \frac{1}{n} P\left(\left|Z^{(1)}\right|>c \varepsilon \sqrt{n}\right)\right] \rightarrow_{\varepsilon \rightarrow 0} 0 .
$$

ii. Let $Z^{(2)}$ be a Hermite random variable of order $q \geq 2$ and $H>1-\frac{1}{2 q}$. Then

$$
\frac{1}{-\log c \varepsilon}\left[\sum_{n \geq 1} \frac{1}{n} P\left(\left|Z_{n}^{(2)}\right|>c \varepsilon n^{1-q(1-H)}\right)-\sum_{n \geq 1} \frac{1}{n} P\left(\left|Z^{(2)}\right|>c \varepsilon n^{1-q(1-H)}\right)\right] \rightarrow_{\varepsilon \rightarrow 0} 0 .
$$

Proof: Let us start with the point i. Assume $H<1-\frac{1}{2 q}$. We can write

$$
\begin{aligned}
& \sum_{n \geq 1} \frac{1}{n} P\left(\left|Z_{n}^{(1)}\right|>c \varepsilon \sqrt{n}\right)-\sum_{n \geq 1} \frac{1}{n} P\left(\left|Z^{(1)}\right|>c \varepsilon \sqrt{n}\right) \\
= & \sum_{n \geq 1} \frac{1}{n}\left[P\left(Z_{n}^{(1)}>c \varepsilon \sqrt{n}\right)-P\left(Z^{(1)}>c \varepsilon \sqrt{n}\right)\right]+\sum_{n \geq 1}\left[\frac{1}{n} P\left(Z_{n}^{(1)}<-c \varepsilon \sqrt{n}\right)-P\left(Z^{(1)}<-c \varepsilon \sqrt{n}\right)\right] \\
\leq & 2 \sum_{n \geq 1} \frac{1}{n} \sup _{x \in \mathbb{R}}\left|P\left(Z_{n}^{(1)}>x\right)-P\left(Z^{(1)}>x\right)\right| .
\end{aligned}
$$


It follows from [10], Theorem 4.1 that

$$
\sup _{x \in \mathbb{R}}\left|P\left(Z_{n}^{(1)}>x\right)-P\left(Z^{(1)}>x\right)\right| \leq c\left\{\begin{array}{l}
\frac{1}{\sqrt{n}}, \quad H \in\left(0, \frac{1}{2}\right] \\
n^{H-1}, \quad H \in\left[\frac{1}{2}, \frac{2 q-3}{2 q-2}\right) \\
n^{q H-q+\frac{1}{2}}, \quad H \in\left[\frac{2 q-3}{2 q-2}, 1-\frac{1}{2 q}\right) .
\end{array}\right.
$$

and this implies that

$$
\sum_{n \geq 1} \frac{1}{n} \sup _{x \in \mathbb{R}}\left|P\left(Z_{n}^{(i)}>x\right)-P\left(Z^{(i)}>x\right)\right| \leq c\left\{\begin{array}{l}
\sum_{n \geq 1} \frac{1}{n \sqrt{n}}, \quad H \in\left(0, \frac{1}{2}\right] \\
\sum_{n \geq 1} n^{H-2}, \quad H \in\left[\frac{1}{2}, \frac{2 q-3}{2 q-2}\right) \\
\sum_{n \geq 1} n^{q H-q-\frac{1}{2}}, \quad H \in\left[\frac{2 q-3}{2 q-2}, 1-\frac{1}{2 q}\right) .
\end{array}\right.
$$

and the last sums are finite (for the last one we use $H<1-\frac{1}{2 q}$ ). The conclusion follows.

Concerning the point ii. (the case $H>1-\frac{1}{2 q}$ ), by using a result in Proposition 3.1 of [2] we have

$$
\sup _{x \in \mathbb{R}}\left|P\left(Z_{n}^{(i)}>x\right)-P\left(Z^{(i)}>x\right)\right| \leq c\left(\mathbf{E}\left|Z_{n}^{(2)}-Z^{(2)}\right|^{2}\right)^{\frac{1}{2 q}} \leq c n^{1-\frac{1}{2 q}-H}
$$

and as a consequence

$$
\sum_{n \geq 1} \frac{1}{n} P\left(\left|Z_{n}^{(2)}\right|>c \varepsilon n^{1-q(1-H)}\right)-\sum_{n \geq 1} \frac{1}{n} P\left(\left|Z^{(2)}\right|>c \varepsilon n^{1-q(1-H)}\right) \leq c \sum_{n \geq 1} n^{-\frac{1}{2 q}-H}
$$

and the above series is convergent because $H>1-\frac{1}{2 q}$.

We state now the Spitzer's theorem for the variations of the fractional Brownian motion.

Theorem 2. Let $f_{1}, f_{2}$ be given by (14), (15) and the constants $c_{1, q, H}, c_{2, q, H}$ be those from Theorem 1.

i. If $0<H<1-\frac{1}{2 q}$ then

$$
\lim _{\varepsilon \rightarrow 0} \frac{1}{\log \left(c_{1, H, q}^{-1} \varepsilon\right)} f_{1}(\varepsilon)=2
$$

ii. If $1>H>1-\frac{1}{2 q}$ then

$$
\lim _{\varepsilon \rightarrow 0} \frac{1}{\log \left(c_{2, H, q}^{-1} \varepsilon\right)} f_{2}(\varepsilon)=\frac{1}{1-q(1-H)}
$$

Proof: It is a consequence of Lemma 1 and Proposition 1 .

Remark 2. Concerning the case $H=1-\frac{1}{2 q}$, note that the correct normalization of $V_{n}$ (3) is $\frac{1}{(\log n) \sqrt{n}}$. Because of the appearance of the term $\log n$ our approach is not directly applicable to this case. 


\section{Hsu-Robbins theorem for the variations of fractional Brown- ian motion}

In this section we prove a version of the Hsu-Robbins theorem for the variations of the fractional Brownian motion. Concretely, we denote here by, for every $\varepsilon>0$

$$
g_{1}(\varepsilon)=\sum_{n \geq 1} P\left(\left|V_{n}\right|>\varepsilon n\right)
$$

if $H<1-\frac{1}{2 q}$ and by

$$
g_{2}(\varepsilon)=\sum_{n \geq 1} P\left(\left|V_{n}\right|>\varepsilon n^{2-2 q(1-H)}\right)
$$

if $H>1-\frac{1}{2 q}$. and we estimate the behavior of the functions $g_{i}(\varepsilon)$ as $\varepsilon \rightarrow 0$. Note that we can write

$$
g_{1}(\varepsilon)=\sum_{n \geq 1} P\left(\left|Z_{n}^{(1)}\right|>c_{1, q, H}^{-1} \varepsilon \sqrt{n}\right), \quad g_{2}(\varepsilon)=\sum_{n \geq 1} P\left(\left|Z_{n}^{(2)}\right|>c_{2, q, H}^{-1} \varepsilon n^{1-q(1-H)}\right)
$$

with $Z_{n}^{(1)}, Z_{n}^{(2)}$ given by (13).

We decompose it as: for $H<1-\frac{1}{2 q}$

$$
\begin{aligned}
g_{1}(\varepsilon) & =\sum_{n \geq 1} P\left(\left|Z^{(1)}\right|>c_{1, q, H}^{-1} \varepsilon \sqrt{n}\right) \\
& +\sum_{n \geq 1}\left[P\left(\left|Z_{n}^{(1)}\right|>c_{1, q, H}^{-1} \varepsilon \sqrt{n}\right)-P\left(\left|Z^{(1)}\right|>c_{1, q, H}^{-1} \varepsilon \sqrt{n}\right)\right]
\end{aligned}
$$

and for $H>1-\frac{1}{2 q}$

$$
\begin{aligned}
g_{2}(\varepsilon) & =\sum_{n \geq 1} P\left(\left|Z^{(2)}\right|>\varepsilon c_{2, q, H}^{-1} n^{1-q(1-H)}\right) \\
& +\sum_{n \geq 1}\left[P\left(\left|Z_{n}^{(2)}\right|>c_{2, q, H}^{-1} \varepsilon n^{1-q(1-H)}\right)-P\left(\left|Z^{(2)}\right|>c_{2, q, H}^{-1} \varepsilon n^{1-q(1-H)}\right)\right]
\end{aligned}
$$

We start again by consider the situation when $Z_{n}^{(i)}$ are replaced by their limits.

Lemma 2. i. Let $Z^{(1)}$ be a standard normal random variable. Then

$$
\lim _{\varepsilon \rightarrow 0}(c \varepsilon)^{2} \sum_{n \geq 1} P\left(\left|Z^{(1)}\right|>c \varepsilon \sqrt{n}\right)=1 .
$$

ii. Let $Z^{(2)}$ be a Hermite random variable with $H>1-\frac{1}{2 q}$. Then

$$
\lim _{\varepsilon \rightarrow 0}(c \varepsilon)^{\frac{1}{1-q(1-H)}} \sum_{n \geq 1} P\left(\left|Z^{(2)}\right|>c \varepsilon n^{1-q(1-H)}\right)=\mathbf{E}\left|Z^{(2)}\right|^{\frac{1}{1-q(1-H)}}
$$


Proof: The part i. is a consequence of the result of Heyde [5]. Indeed take $X_{i} \sim N(0,1)$ in (1). Concerning part ii. we can write

$$
\begin{aligned}
& \lim _{\varepsilon \rightarrow 0}(c \varepsilon)^{\frac{1}{1-q(1-H)}} \sum_{n \geq 1} \Phi_{Z^{(2)}}\left(c \varepsilon n^{1-q(1-H)}\right) \\
= & \lim _{\varepsilon \rightarrow 0}(c \varepsilon)^{\frac{1}{1-q(1-H)}}\left[\int_{1}^{\infty} \Phi_{Z^{(2)}}\left(c \varepsilon x^{1-q(1-H)}\right) d x-\int_{1}^{\infty} P_{1}(x) d\left[\Phi_{Z^{(2)}}\left(c \varepsilon x^{1-q(1-H)}\right)\right]\right] \\
& :=\lim _{\varepsilon \rightarrow 0}(A(\varepsilon)+B(\varepsilon))
\end{aligned}
$$

with $P_{1}(x)=[x]-x+\frac{1}{2}$. Moreover

$$
\begin{aligned}
A(\varepsilon) & =(c \varepsilon)^{\frac{1}{1-q(1-H)}} \int_{1}^{\infty} \Phi_{Z^{(2)}}\left(c \varepsilon x^{1-q(1-H)}\right) d x \\
& =\frac{1}{1-q(1-H)} \int_{c \varepsilon}^{\infty} \Phi_{Z^{(2)}}(y) y^{\frac{1}{1-q(1-H)}-1} d y .
\end{aligned}
$$

Since $\Phi_{Z^{(2)}}(y) \leq y^{-2}$ we have $\Phi_{Z^{(2)}}(y) y^{\frac{1}{1-q(1-H)}} \rightarrow_{y \rightarrow \infty} 0$ and therefore

$$
A(\varepsilon)=-\Phi_{Z^{(2)}}(c \varepsilon)(c \varepsilon)^{\frac{1}{1-q(1-H)}}-\int_{c \varepsilon}^{\infty} \Phi_{Z^{(2)}}^{\prime}(y) y^{\frac{1}{1-q(1-H)}} d y
$$

where the first terms goes to zero and the second to $\mathbf{E}\left|Z^{(2)}\right|^{\frac{1}{1-q(1-H)}}$. The proof that the term $B(\varepsilon)$ converges to zero is similar to the proof of Lemma 2 , point ii.

Remark 3. The Hermite random variable has moments of all orders (in particular the moment of order $\frac{1}{1-q(1-H)}$ exists) since it is the value at time 1 of a selfsimilar process with stationary increments.

Proposition 2. i. Let $H<1-\frac{1}{2 q}$ and let $Z_{n}^{(1)}$ be given by (13). Let also $Z^{(1)}$ be a standard normal random variable. Then

$$
(c \varepsilon)^{2} \sum_{n \geq 1}\left[P\left(\left|Z_{n}^{(1)}\right|>c \varepsilon \sqrt{n}\right)-P\left(\left|Z^{(1)}\right|>c \varepsilon \sqrt{n}\right)\right] \rightarrow_{\varepsilon \rightarrow 0} 0
$$

ii. Let $H>1-\frac{1}{2 q}$ and let $Z_{n}^{(2)}$ be given by (13). Let $Z^{(2)}$ be a Hermite random variable. Then

$$
(c \varepsilon)^{\frac{1}{1-q(1-H)}} \sum_{n \geq 1}\left[P\left(\left|Z_{n}^{(2)}\right|>c \varepsilon n^{1-q(1-H)}\right)-P\left(\left|Z^{(2)}\right|>c \varepsilon n^{1-q(1-H)}\right)\right] \rightarrow_{\varepsilon \rightarrow 0} 0 .
$$

Remark 4. Note that the bounds (16), (18) does not help here because the series that appear after their use are not convergent. 
Proof of Proposition 2: Case $H<1-\frac{1}{2 q}$. We have, for some $\beta>0$ to be chosen later,

$$
\begin{aligned}
& \varepsilon^{2} \sum_{n \geq 1}\left[P\left(\left|Z_{n}^{(1)}\right|>c \varepsilon \sqrt{n}\right)-P\left(\left|Z^{(1)}\right|>c \varepsilon \sqrt{n}\right)\right] \\
= & \varepsilon^{2} \sum_{n=1}^{\left[\varepsilon^{-\beta}\right]}\left[P\left(\left|Z_{n}^{(1)}\right|>c \varepsilon \sqrt{n}\right)-P\left(\left|Z^{(1)}\right|>c \varepsilon \sqrt{n}\right)\right] \\
& +\varepsilon^{2} \sum_{n>\left[\varepsilon^{-\beta}\right]}\left[P\left(\left|Z_{n}^{(1)}\right|>c \varepsilon \sqrt{n}\right)-P\left(\left|Z^{(1)}\right|>c \varepsilon \sqrt{n}\right)\right] \\
:= & I_{1}(\varepsilon)+J_{1}(\varepsilon) .
\end{aligned}
$$

Consider first the situation when $H \in\left(0, \frac{1}{2}\right]$. Let us choose a real number $\beta$ such that $2<\beta<4$. By using (16),

$$
I_{1}(\varepsilon) \leq c \varepsilon^{2} \sum_{n=1}^{\left[\varepsilon^{-\beta}\right]} n^{-\frac{1}{2}} \leq c \varepsilon^{2} \varepsilon^{-\frac{\beta}{2}} \rightarrow_{\varepsilon \rightarrow 0} 0
$$

since $\beta<4$. Next, by using the bound for the tail probabilities of multiple integrals and since $\mathrm{E}\left|Z_{n}^{(1)}\right|^{2}$ converges to 1 as $n \rightarrow \infty$

$$
\begin{aligned}
& J_{1}(\varepsilon)=\varepsilon^{2} \sum_{n>\left[\varepsilon^{-\beta}\right]} P\left(Z_{n}^{(1)}>c \varepsilon \sqrt{n}\right) \leq c \varepsilon^{-2} \sum_{n>\left[\varepsilon^{-\beta}\right]} \exp \left(\frac{-c \varepsilon \sqrt{n}}{\left(\mathrm{E}\left|Z_{n}^{(1)}\right|^{2}\right)^{\frac{1}{2}}}\right)^{\frac{2}{q}} \\
& \leq \varepsilon^{2} \sum_{n>\left[\varepsilon^{-\beta}\right]} \exp \left(\left(-c n^{\left.-\frac{1}{\beta} \sqrt{n}\right)^{\frac{2}{q}}}\right)\right.
\end{aligned}
$$

and since converges to zero for $\beta>2$. The same argument shows that $\varepsilon^{2} \sum_{n>\left[\varepsilon^{-\beta}\right]} P\left(Z^{(1)}>c \varepsilon \sqrt{n}\right)$ converges to zero.

The case when $H \in\left(\frac{1}{2}, \frac{2 q-3}{2 q-2}\right)$ can be obtained by taking $2<\beta<\frac{2}{H}$ (it is possible since $H<1$ ) while in the case $H \in\left(\frac{2 q-3}{2 q-2}, 1-\frac{1}{2 q}\right)$ we have to choose $2<\beta<\frac{2}{q H-q+\frac{3}{2}}$ (which is possible because $H<1-\frac{1}{2 q}$ !).

Case $H>1-\frac{1}{2 q}$. We have, with some suitable $\beta>0$

$$
\begin{aligned}
& \varepsilon^{\frac{1}{1-q(1-H)}} \sum_{n \geq 1}\left[P\left(\left|Z_{n}^{(2)}\right|>c \varepsilon n^{1-q(1-H)}\right)-P\left(\left|Z^{(2)}\right|>c \varepsilon n^{1-q(1-H)}\right)\right] \\
= & \varepsilon^{\frac{1}{1-q(1-H)}} \sum_{n=1}^{\left[\varepsilon^{-\beta}\right]}\left[P\left(\left|Z_{n}^{(2)}\right|>c \varepsilon n^{1-q(1-H)}\right)-P\left(\left|Z^{(2)}\right|>c \varepsilon n^{1-q(1-H)}\right)\right] \\
& +\varepsilon^{\frac{1}{1-q(1-H)}} \sum_{n \geq\left[\varepsilon^{-\beta}\right]}\left[P\left(\left|Z_{n}^{(2)}\right|>c \varepsilon n^{1-q(1-H)}\right)-P\left(\left|Z^{(2)}\right|>c \varepsilon n^{1-q(1-H)}\right)\right] \\
:= & I_{2}(\varepsilon)+J_{2}(\varepsilon) .
\end{aligned}
$$

Choose $\frac{1}{1-q(1-H)}<\beta<\frac{1}{(1-q(1-H))\left(2-H-\frac{1}{2 q}\right)}$ (again, this is always possible when $H>1-\frac{1}{2 q}$ !). Then

$$
I_{2}(\varepsilon) \leq c e^{\frac{1}{1-q(1-H)}} \varepsilon^{(-\beta)\left(2-H-\frac{1}{2 q}\right)} \rightarrow_{\varepsilon \rightarrow 0} 0
$$


and by (9)

$$
J_{2}(\varepsilon) \leq c \sum_{n>\left[\varepsilon^{-\beta}\right]} \exp \left(\left(\frac{-c \varepsilon n^{1-q(1-H)}}{\left(\mathrm{E}\left|Z_{n}^{(2)}\right|^{2}\right)^{\frac{1}{2}}}\right) \leq c \sum_{n>\left[\varepsilon^{-\beta}\right]} \exp \left(c n^{-\frac{1}{\beta}} n^{1-q(1-H)}\right)^{\frac{2}{q}} \rightarrow_{\varepsilon \rightarrow 0} 0\right.
$$

We state the main result of this section which is a consequence of Lemma 2 and Proposition 2 .

Theorem 3. Let $q \geq 2$ and let $c_{1, q, H}, c_{2, q, H}$ be the constants from Theorem 1. Let $Z^{(1)}$ be a standard normal random variable, $Z^{(2)}$ a Hermite random variable of order $q \geq 2$ and let $g_{1}, g_{2}$ be given by (19) and (20). Then

i. If $0<H<1-\frac{1}{2 q}$, we have $\left(c_{1, q, H}^{-1} \varepsilon\right)^{2} g_{1}(\varepsilon) \rightarrow_{\varepsilon \rightarrow 0} 1=\mathbf{E} Z^{(1)}$.

ii. If $1-\frac{1}{2 q}<H<1$ we have $\left(c_{2, q, H}^{-1} \varepsilon\right)^{\frac{1}{1-q(1-H)}} g_{2}(\varepsilon) \rightarrow_{\varepsilon \rightarrow 0} \mathbf{E}\left|Z^{(2)}\right|^{\frac{1}{1-q(1-H)}}$.

Remark 5. In the case $H=\frac{1}{2}$ we retrieve the result (1) of [5]. The case $q=1$ is trivial, because in this case, since $V_{n}=B_{n}$ and $\mathbf{E} V_{n}^{2}=n^{2 H}$, we obtain the following (by applying Lemma 1 and 2 with $q=1$ )

$$
\frac{1}{\log \varepsilon} \sum_{n \geq 1} \frac{1}{n} P\left(\left|V_{n}\right|>\varepsilon n^{2 H}\right) \rightarrow_{\varepsilon \rightarrow 0} \frac{1}{H}
$$

and

$$
\varepsilon^{2} \sum_{n \geq 1} P\left(\left|V_{n}\right|>\varepsilon n^{2 H}\right) \rightarrow_{\varepsilon \rightarrow 0} \mathbf{E}\left|Z^{(1)}\right|^{\frac{1}{H}} .
$$

Remark 6. Let $\left(\varepsilon_{i}\right)_{i \in \mathbb{Z}}$ be a sequence of i.i.d. centered random variable with finite variance and let $\left(a_{i}\right)_{i \geq 1}$ a square summable real sequence. Define $X_{n}=\sum_{i \geq 1} a_{i} \varepsilon_{n-i}$. Then the sequence $S_{N}=$ $\sum_{n=1}^{N}\left[K\left(X_{n}\right)-\mathbf{E} K\left(X_{n}\right)\right]$ satisfies a central limit theorem or a non-central limit theorem according to the properties of the measurable function $K$ (see [6] or [14]). We think that our tools can be applied to investigate the tail probabilities of the sequence $S_{N}$ in the spirit of [5] or [12] at least the in particular cases (for example, when $\varepsilon_{i}$ represents the increment $W_{i+1}-W_{i}$ of a Wiener process because in this case $\varepsilon_{i}$ can be written as a multiple integral of order one and $X_{n}$ can be decomposed into a sum of multiple integrals. We thank the referee for mentioning the references [6] and [14].

\section{References}

[1] L.M. Albin, A note on Rosenblatt distributions. Statist. Probab. Lett., 40(1) (1998), 83-91.

[2] J.-C. Breton and I. Nourdin, Error bounds on the non-normal approximation of Hermite power variations of fractional Brownian motion. Electronic Communications in Probability, 13 (2008), 482-493.

[3] P. Erdös, On a theorem of Hsu and Robbins. Ann. Math. Statistics, 20(1949), 286-291. 
[4] P. Erdös, Remark on my paper "On a theorem of Hsu and Robbins". Ann. Math. statistics, 21 (1950), 138.

[5] C.C. Heyde, A supplement to the strong law of large numbers. Journal of Applied Probability, 12(1975), 173-175.

[6] H-C. Ho and T. Hsing, Limit theorems for functionals of moving averages. The Annals of Probability, 25(4) (1997), 1636-1669.

[7] P. Hsu and H. Robbins, Complete convergence and the law of large numbers. Proc. Nat. Acad. Sci. U.S.A., 33(1947), 25-31.

[8] P. Major, Tail behavior of multiple integrals and U-statitics. Probability Surveys, 2 (2005), 448-505.

[9] I. Nourdin, D. Nualart and C.A. Tudor, Central and non-central limit theorems for weighted power variations of fractional Brownian motion (2008), Preprint.

[10] I. Nourdin and G. Peccati, Stein's method on Wiener chaos (2007), To appear in Probability Theory and Related Fields.

[11] D. Nualart, Malliavin Calculus and Related Topics. Second Edition (2006) Springer.

[12] A. Spataru, Precise Asymptotics in Spitzer's law of large numbers. Journal of Theoretical Probability, 12(3)(1999), 811-819.

[13] F. Spitzer, A combinatorial lemma and its applications to probability theory. Trans. Amer. Math. Soc., 82(1956), 323-339.

[14] W. B. Wu, Unit root testing for functionals of linear processes. Econometric Theory, 22(1)(2006), 1-14. 